\title{
Antagonismos Ideológicos en la Fundación del Convento la Asunción de María en Yautepec, Mor. \\ Rodolfo Abarca Díaz ${ }^{\text {a, } 1^{*}}$
}

a Sistema de Estudios de Posgrado e Investigación, Facultad de Arquitectura, Universidad Autónoma del Estado de Morelos.

\begin{abstract}
Resumen
La presente investigación aborda el tema de la Cosmovisión Prehispánica y su permanencia aún vigente en el sitio elegido por los primeros evangelizadores en la Nueva España hasta la promulgación de las ordenanzas de Felipe II. El caso de estudio es el Convento Dominico La Asunción de María, en el municipio morelense de Yautepec. La razón de esta elección radica en que, el convento de la Asunción de Maria en Yautepec, Morelos no se edificó sobre las ruinas del teocalli local (Smith, 1994, 1997, 2006), por lo tanto es probable que la elección del terreno obedecería a factores de reminiscencia en el imaginario colectivo de los indígenas conquistados e involucrados en su construcción, es decir, este edificio representa un caso singular ya que no obedece a la lógica urbana peninsular vigente en el siglo XVI. Lógica en la cual era más práctico utilizar como cimiento la base anterior y reutilizar sus piedras en el nuevo templo cristiano (McAndrew, 1963; Kubler, 2009; Weckmann, 1996; Sprajc, 2001). Por lo tanto la presente investigación tiene como objetivo demostrar la persistencia de la cosmovisión prehispánica en la refundación de la villa de Yautepec y la construcción del convento dominico la Asunción de Maria; su justificación consiste en llenar un vacío histórico. La metodología empleada consistió en la observación de campo, revisión de la bibliografía al alcance e interpretación de datos. Las corrientes de pensamiento analizadas son: la cosmovisión prehispánica y los procesos de refundación novo hispana del siglo XVI hasta 1573.
\end{abstract}

Palabras Clave: cosmovisión prehispánica, refundaciones urbanas, sincretismos urbanos, sincretismos arquitectónicos.

\section{Introducción}

Con la presente investigación se pretende demostrar que la construcción de algunos de los nuevos templos cristianos no sólo obedeció a factores prácticos de edificación, sino que en tal acto persistieron en los indígenas meta representaciones sacras y rituales, mismas que no eran desconocidas para los frailes evangelizadores pues existieron concesiones al respecto, tal cual lo plantea García Zambrano (García Zambrano, 2004:60) en la fundación de un "Pueblo Nuevo". Con ello, surgen los "Sincretismos Urbanos".

Nuestro objetivo principal es evidenciar la permanencia de las meta representaciones sacras y rituales en la construcción de un convento del siglo XVI, tomando en cuenta que el Convento Mariano de Yautepec no se construyó sobre un teocalli, y sin embargo conservó el alineamiento prehispánico hacia tres elementos en el paisaje inmediato. Se demostrará que existió una transferencia de estos tres elementos, atemaxac o atemajac, rinconadas o joyas y los macizos rocosos, en el imaginario identitario colectivo de indígenas de la generación posterior a la Conquista.

Nuestra hipótesis de trabajo es: en la construcción del convento Dominico del siglo XVI La Asunción de María en Yautepec, Morelos, se conservaron orientaciones prehispánicas hacia elementos en el paisaje inmediato y mediato, orientaciones recurrentes en la arquitectura y el urbanismo prehispánico de Mesoamérica; destacando que el convento la Asunción de María en Yautepec, Morelos es un edificio posterior a la segunda mitad del siglo XVI, y que sui generis no se construyó sobre un teocalli. Por lo tanto es probable la transferencia de la meta representacional de la cosmovisión prehispánica y en particular del "eje cósmico" en la elección del terreno para edificar el convento.

\section{Materiales y Métodos}

A Desde el 2013 se han realizado indagaciones sobre el tema, como se evidencia en un trabajo de investigación anterior en el que se planteó un eje de trazo geométrico (Abarca, 2015: 60) y se involucran tres elementos en el paisaje; estos accidentes naturales fueron considerados sacros y rituales por los pobladores de Mesoamérica; el atemaxac o atemajac, las rinconadas o joyas, y los macizos rocosos. En esta investigación se agrega el templo cristiano, el cual queda inmerso en el centro de esta triada geográfica sagrada. A partir del 2013 se han realizado recorridos de campo, levantamientos topográficos y arquitectónicos; además de observaciones astronómicas, principalmente en las fechas cercanas al 15 de agosto, así como observaciones al horizonte inmediato y mediato; todo esto acompañado de la revisión bibliográfica al alcance. Ya recopilada esta información se analizó e interpretó, los resultados y conclusiones son nuestra aportación al campo de estudio.

De acuerdo a George Kubler (2009), la construcción del Convento de Yautepec se atribuye a fray Lorenzo de la Asunción; el historiador plantea la hipótesis de que Yautepec fue una visita no registrada de Totolapan hasta la década de 1540; plantea que era un edificio de pequeñas dimensiones que no se encontraba ocupado; la construcción formal del convento inicia hacia 1554. El autor sostiene esta hipótesis de

\footnotetext{
* Autor en correspondencia.

Correo electrónico: arq_abarka@icloud.com
} 
la siguiente manera: 1) la nave cuenta con una estereotomía de la piedra mucho más elaborada que en la primera mitad del siglo XVI; 2) la construcción del claustro se realizó aún con piedra bruta (Kubler: 2009).

Mario Camacho Cardona (2009), atribuye una primera etapa constructiva hacia 1535 , una segunda y última entre 1560/70 al 76; el programa arquitectónico constaba de atrio, capilla de indios, iglesia, bautisterio, claustro alto y bajo, dormitorios, celdas y huerta (Camacho, 2009). Actualmente se puede ver inscrito en la fachada poniente un 1567, que probablemente sea referencia sobre el año de culminación de la obra.

Abarca (2015) demostró que el convento dominico no fue el primer edificio religioso cristiano en Yautepec; la iglesia de San Juan, a unos 500 metros al sur del actual convento, es la iglesia primigenia del sitio.

\subsection{Cosmovisión prehispánicas}

De acuerdo a Laura Ledesma Gallegos (2012) en el urbanismo mesoamericano el paisaje fue el factor determinante en la búsqueda del lugar propicio para fundar y asentarse definidamente como un pueblo. El territorio elegido debería de tener las condiciones necesarias tanto para conseguir el sustento como para tener dónde realizar los rituales, ambos aspectos para la subsistencia de sus futuros habitantes: suelo fértil, aguas verdiazules y el receptáculo que contenía todos los mantenimientos, esto es un macizo rocoso, ya sea montaña o cerro (Ledesma, 2012). Alfredo López Austin (1994) manifiesta que el sitio elegido también debería de rememorar el origen mítico y común del que habían surgido todos los pueblos (López, 1994). Según Ángel Julián García Zambrano (2000), en el asiento de los pueblos indígenas debía existir una fisiografía natural específica, la cual tendría que estar conformada por cerros confinantes de fuentes acuáticas o ubicadas cerca de ellas (García Zambrano 2000: 35).

La montaña solía ser el eje rector que determinaba la traza urbana del asentamiento, así mismo marcaba la distribución espacial de los componentes arquitectónicos del pueblo. De esta forma la pirámide hecha por el hombre era una representación de la montaña hecha a mano que se repetía reiterativamente en el paisaje urbano (Ledesma, 2012). James Lockhart (1999) determina que la montaña y la pirámide son símbolos del pueblo que confirman la pertenencia a un territorio y la soberanía del pueblo; y como tal permanecen en la memoria colectiva de la comunidad indígena, aun después de la Conquista (Lockhart, 1999). Ismael Arturo Montero García (2001) propone que elementos naturales como las montañas o volcanes tuvieron tal importancia en la cosmogonía indígena que los antiguos habitantes imitaron su forma. De allí que en las relaciones geográficas del siglo XVI se les identifica como "la montaña hecha a mano". En este sentido se propone observar a la pirámide como el arquetipo de la montaña visualizada en el interior del espacio urbano; ambos elementos cuentan con componentes de culto en común: la montaña tiene adoratorios que pueden ser cuevas o cañadas; y la pirámide está rematada por el templo, ambos elementos, uno natural y otro artificial tienen la función de ser adoratorios (Montero, 2001: 13-22).

Las montañas o cerros también cumplían con la función de marcadores de tiempos. Las culturas prehispánicas desarrollaron métodos de observación para llevar el seguimiento del tiempo, utilizando como referencia la posición de algunos astros con la relación orográfica de su entorno. Así es como surgieron los calendarios de horizonte que servían no sólo de guías visuales del transcurrir del tiempo, sino también como instrumentos destinados a calibrar la concordancia del movimiento aparente de los astros con su calendario. Por citar un ejemplo, Jesús Galindo Trejo y César Esteban López (2001) plantean que el Cerro de San Miguel es un posible marcador calendárico astronómico del sitio preclásico de Cuicuilco (Trejo y López, 2001). Como se explica en los párrafos siguientes, la observación de la naturaleza por parte del hombre mesoamericano no sólo fue para asegurar el abastecimiento de alimentos a través de su atención a los ciclos agrícolas, sino que también establecía un marco organizativo para toda la sociedad en forma de un sistema calendárico que indicara la correcta sucesión de los ritos religiosos, alrededor de los cuales estaba organizada la sociedad.

De acuerdo a García Zambrano (2000) a los macizos rocosos se suman las "rinconadas" o "rincones" como parte de la geografía sagrada prehispánica, este tipo de ambiente se distingue por situarse en una depresión natural rodeada de cerros o montañas, susceptible a ser inundada por excedentes hídricos provenientes de afluentes o veneros que brotaban de las cuevas sagradas cercanas. Estos elementos geográficos podían ser de grandes dimensiones o bien ser pequeños microambientes encañonados, o simples parcelas situadas junto a barrancas, o incluso sobre las mismas faldas del terreno. Según el mismo autor, dentro de las inmediaciones de la rinconada habitaban familias de manera dispersa, de tal forma que no ejercían mayor presión extractiva sobre el territorio (García Zambrano, 2000). Para concluir este apartado se hace referencia a Bernal-García (2005), quien afirma que para las culturas mesoamericanas la planeación urbana implicaba la creación de una obra de arte como tal (Bernal-García, 2004).

Para conocer el proceso de urbanización temprana en nuestro edificio caso de estudio, juzgamos pertinente conocer cómo sucedió este hecho en la Nueva España durante la época de contacto y el subsecuente siglo XVI temprano. Como preámbulo y de acuerdo a García Zambrano (2000) se reconoce que los europeos tuvieron gran dificultad para comprender la visión de los indígenas acerca de la naturaleza. Este impedimento residía básicamente en la manera de observar el territorio, los europeos estaban ideológicamente limitados para entender el concepto ritual de los nativos, quienes veían en el entorno elementos de mantenimiento y sacralidad. Esta percepción incitaba a los indígenas a respetar la naturaleza, y sólo tomar de ella lo suficiente para su supervivencia (García Zambrano, 2000). El autor citado evidencia la actualidad del culto al paisaje circundante en algunas comunidades náhua parlantes de Veracruz y Puebla.

En las relaciones geográficas de Michoacán de Rene Acuña (Acuña, 1987), citadas por García Zambrano (2000), se describe la perspectiva de los europeos sobre que los naturales vivían dispersos por el territorio, organizados en núcleos familiares, cada padre con su mujer e hijos; el documento del siglo XVI evidencia que: Hernando de Alvarado por consigna del Virrey Antonio de Mendoza, sometió a los indígenas para ser reubicados, de tal manera que los curas y religiosos tuvieran la tarea de evangelización más fácil y práctica, este reacomodo costó la vida de muchos indígenas (García Zambrano, 2000). En la elevada tasa de mortandad experimentada por los indígenas influye la tristeza sufrida por 
el desarraigo a su lugar primigenio, los europeos estaban totalmente impedidos para comprender la sacralidad del entorno, éstos sólo tenían una visión occidental de la naturaleza, es decir, la explotación de recursos era lo primordial y de ello dependía la elección del sitio a ocupar y urbanizar.

El eje cósmico planteado por López Austín (2008) se incorpora a nuestra discusión con la premisa de: "Lo que es en el cielo, es en la tierra". De acuerdo al autor los antiguos nahuas hablaron de tres pisos cósmicos: chicnauhtopan. Son vastas las representaciones visuales, la más detallada muestra sus componentes. Sobre el plano terrestre se eleva el Monte Sagrado, en la cumbre del monte se yergue el árbol florido, bajo el monte se encuentra el acuático y frío lugar de la muerte (López, 2008).

\section{Resultados y Discusión}

En el siguiente apartado se aplican las meta representaciones descritas de manera sucinta y se analiza el sitio donde se edificó el Convento en Yautepec, así como el edificio mismo.

El presente análisis demuestra la relación de geometría y posicionamiento del edificio con el paisaje inmediato; la construcción se ubica en la confluencia de dos afluentes de agua: el río Yautepec que es de cauce continuo, y la Barranca Apanquetzalco de cauce temporal; accidente geográfico recurrente en las meta representaciones sacralizadas por los antiguos nahuas, el sitio hace referencia a una entrepierna, en donde brota líquido amniótico, lugar dotado de la más alta sacralidad pues es de ahí de donde surge vida (García Zambrano, 2006).

Sobre un plano conceptual se observa la colocación del convento que se construyó en una zona cargada de meta representaciones sacralizadas por los indígenas; el atemaxac, atemajac o atlamaxac, fue el marco idóneo en la elección de los Dominicos; al oriente del convento y al pie del Cerro Tenayo se localizaba una cueva, esta oquedad se encuentra documentada en la relación geográfica de Tepoztlán (Acuña, 1984). En la actualidad la "Cueva" sólo persiste en el imaginario colectivo local.

$\mathrm{Al}$ sur del conjunto conventual se localizaba un manantial, el cual según nuestro informante Agustín Abarca Jiménez, fue tapado en 1985 cuando se construyeron los gaviones del río (Abarca Jiménez, 2016: comentario personal y enseñanzas); al oriente, se localiza el Tenayo, un macizo, cerro primigenio que prestó su ubicación y morfología al imaginario prehispánico y cargó de misticismo el sitio, Altépetl y Tonacatépetl de lugar. Es tanta la carga sacra del cerro, que es ahí en donde mora Yiauhtécatl, "uno de los cuatrocientos" (de Sahagún, 2000: 1350).

En el entorno inmediato al convento se localizan cuatro elementos geográficos de altísima estima sacra prehispánica que fueron del conocimiento de los evangelizadores, quienes es muy probable aceptaran la persistencia de estos elementos en la edificación de su conjunto.

El siguiente análisis pretende mostrar la relación del convento con elementos territoriales en una escala mayor a los que se adhieren otros elementos. Al poniente, a la zona al pie del Cerro de "las Tetillas" se le conoce como Coatlán Chico, ahí se localizan tres elementos naturales y uno de construcción humana.
A un horizonte de $1300 \mathrm{msnm}$ se localizan las piedras de Coatlán o piedras de los Reyes, cuentan con petrograbados, elementos del Periodo Posclásico que hacen alusión a la creación del calendario, en una clara representación de Oxomoco y Cipactonal, documentados por Cecilio Robelo en 1910 (Robelo, 1912: 9), la hipótesis del historiador se comprueba al analizar la lámina 3 del Códice Borbónico; al norte de los petrograbados se localiza una cueva o resumidero, la zona es una joya o depresión natural a unos $1280 \mathrm{msnm}$.

Una vez que se localizaron los elementos se analizó en la cartografía del INEGI un posible trazo o eje que uniera las piedras grabadas con el atemaxac, posteriormente se ratificó con un levantamiento en campo; los resultados fueron reveladores, el eje planteado tiene una desviación con el eje oriente poniente de 17.5 grados 23 , en sentido contrario a las manecillas del reloj, tomando como referencia el norte astronómico obtenido en campo; aún más revelador es el siguiente hallazgo, el eje de trazo "hipotético" se sobrescribe no solo de las piedras al atemaxac, teniendo las siguientes coincidencias en su desarrollo geométrico, inicia en los petrograbados, sigue el trazo casi perfecto del "camino viejo", corta el atemaxac, divide en dos la nave principal del convento, pasando por el ábside, toca el punto en donde según las relaciones geográficas de Tepoztlán se encontraba una cueva y culmina en la cima del Cerro Tenayo. Es probable que al continuar el eje o trazo hipotético al paisaje mediato, se encuentren alineaciones con el Volcán Popocatépetl; se están haciendo observaciones y mediciones pertinentes para demostrar esta premisa.

En una investigación anterior (Abarca, 2015) se determinó que la orientación del muro sur del Tecpan es de $17.5^{\circ}$, por consiguiente encaja en el de la familia de $17^{\circ}$ desarrollado por Ponce de León 1983; Aveni, 1996; Galindo, 1994; Broda, 1993, 1997, 2001, 2003; Sprajc 2001. Por consiguiente el talud sur y el camino viejo son parcialmente paralelos, en el desarrollo de la tesis doctoral se determinará si en la confluencia de ambas líneas imaginarias, se presenta un elemento paisajístico de importancia sacra.

En la presente investigación también se determinó que el horizonte de los petrograbados y la cima del Tenayo están a $1300 \mathrm{msnm}$ y $1303 \mathrm{msnm}$, respectivamente. Por lo tanto es probable que el Cerro Tenayo fuera el punto de observación para determinar las alineaciones y orientaciones del trazado urbano y edificios tanto prehispánicos como novohispanos.

Por último, se observó la puesta del sol en distintas fechas representativas, observando que el día 15 de agosto el disco solar se alinea con el muro sur de la nave principal, ocultándose en el Cerro de "las Tetillas" en una alineación casi perfecta con las piedras de Coatlán.

23 Las orientaciones de $17^{\circ}$ han sido ampliamente desarrolladas por un grupo especializado de arqueoastrónomos, que señala la puesta del Sol en los días cercanos al 13 de agosto. (Ponce de León 1983; Aveni, 1991: 263; Galindo, 1994: 129; Peeler 1989; Broda, 1993: 261; Sprajc 1990; 1995, 2001).

Cabe destacar que el día que se celebra la festividad de la Asunción de María, es precisamente el 15 de agosto. Sprajc (2001) señala que los edificios prehispánicos con orientaciones de $17^{\circ}$ señalan la puesta del sol en días cercanos al 13 de agosto (Sprajc, 2001).

\section{Conclusiones}


El convento dominico La Asunción de María de Yautepec, Morelos, es una construcción conventual muy tardía que se edificó dos generaciones después de la conquista militar; está comprobado que no fue el primer edificio religioso de la zona (Abarca, 2015: 111), no se edificó sobre restos de un anterior teocalli o tecpan tlahuica/mexica, sin embargo, presenta características típicas de edificaciones prehispánicas.

Se localiza en una confluencia de dos fuentes acuáticas, lugar denominado como entrepierna de la tierra, atemaxac, atemajac o atlamaxac; un lugar con una vasta carga ritual para los nahuas del altiplano central. En esta horcajadura se localizan accidentes geográficos también ritualizados por los anteriores habitantes: cueva, cerro, manantial y meandro. En un paisaje mediato cercano se ubican otros elementos prehispánicos importantísimos: una joya, una cueva o resumidero, y petrograbados.

La unión de estos elementos con lo que determinamos "eje de trazo" hace referencia a lo que López Austin (2008) denomina "eje cósmico" (López, 2008). Y la evidente puesta del sol el día 15 de agosto que se alinea con el muro sur de la nave principal, ratifica la persistencia de meta representaciones sacras y rituales en la elección del sitio y la construcción del convento mismo.

Por lo tanto podemos afirmar que en este edificio analizado existen sincretismos urbanos, en el imaginario colectivo sobre la geografía y el cosmos sagrados, persistieron en la edificación del convento; podemos afirmar de manera hipotética que este acto no fue del desconocimiento de los evangelizadores. Como parte de un trabajo más extenso se pretende demostrar que estos sincretismos están presentes en otras localidades; lo cual se demostrará en la tesis doctoral.

\section{English Summary}

Ideological antagonisms in the foundation of the Asunción de María Convent in Yautepec Morelos

\footnotetext{
Abstract

This work consists of a historical and architectural investigation of the church of San Nicolás Tolentino of the city of Taxco de Alarcón state of Guerrero, whose objective was to discover the historical and symbolic content of such a humble monument. It is one of the many churches built by the first evangelizers of the region: the mendicant friars of the order of San Francisco, they arrived in Cuernavaca in 1525. The same, following the rules of poverty observed for almost three hundred years, they made it from a single nave, with walls of stone masonry seated with mud, in the prehispanic way and with roof of palm to two waters. And despite its marked simplicity, it seems to be a true "palimpsest", where you can clearly read, among other things, three adaptations that through its history were carried out: The first whose factory was in charge by the parents Franciscans in the sixteenth century, the second corresponding to the eighteenth century, and the third to the second half of the nineteenth century. In another sense, it can be said that, the geometric arrangement of the architectural plan of the building, obeyed the canons of proportionality and symbolism of the first churches of Christianity. Not so its location and orientation, since these characteristics do not seem to correspond to the
}

European own traditions, but rather, to a typical context of the "Pre-Hispanic Ritual Landscape". Therefore, it is desirable that knowledge of the attributes of the church of San Nicolás Tolentino brought to light, help to justify their permanence in an increasingly changing urban environment.

\section{Keywords:}

Robust control, multiobjective optimization, Physical Programming, Genetic Algorithms

\section{Referencias}

Aveny, Anthony (1991), Observadores del cielo en el México antiguo, F. C. E. 1991, México. Babini Baan, Andrea (2005), Taxco de Alarcón, Universidad Autónoma de Guerrero, México. Benavente, Fray Toribio de (1996), Historia de los indios de la Nueva España, edit.

Porrúa, México.

Bustamante Acuña (2007), Manuel Forma y espacio, Universidad Iberoamericana, México. Centini, Massimo (2004), El simbolismo esotérico, Editorial de Vecchi, Barcelona.

Abarca Díaz, R. (2015). Tesis inédita: Centro urbano primigenio de Yautepec, Mor. Cuernavaca, México: Universidad Autónoma del Estado de Morelos.

Acuña, R. (1987). Relaciones Geográficas del Siglo XVI: MIchoacán. Cd. de México: Instituto de Investigaciones Antropológicas, Universidad Nacional Autónoma de México.

Aveni F., A. (1996). The Mayan Number of Time: Intervalic Time Reckoning in the Mayan Codices, part. I. Archaeoastronomy, núm. 20, 32 .

Bernal-García, M. (2004). Cholollan: de montaña a topónimo de paraje y ciudad. En L. Noelle (Ed.), La ciudad: problema integral de preservación patrimonial (págs. 103-124). Cd. de México, México: Universidad Nacional Autónoma de México, Instituto de Investigaciones Estéticas.

Broda, J. (1983). Ciclos agrícolas en el culto: un problema de la correlación del calendario mexica. En

A. Aveni, \& G. Brotherston (Edits.), Calendars in Mesoamerica and Peru: Native American Computations of time (págs. 145-165). Oxford: Oxford, BAR. International.

Broda, J. (1997). El culto mexica de los cerros de la cuenca de México. En B. Albores, \& J. Broda (Edits.), Graniceros, cosmovisión y meteorología indígena de Mesoamérica (págs. 49-90). Cd. de México, México: Universidad Nacional Autónoma de México, El Colegio Mexiquense A.C

Broda, J. (2001). Ritos mexicas en los cerros de la cuenca: los sacrificios de niños. En J. Broda, S. Iwaniszeski, \& A. Montero (Edits.), La montaña en el paisaje ritual (págs. 296-329). Cd. de México, México: Universidad Nacional Autónoma de México.

Broda, J. (2003). El culto mexica de los cerros de la cuenta de México: apuntes para la discusión de graniceros. En B. Albores, \& J. Broda (Edits.), Graniceros: cosmovisión y meteorología

Camacho Cardona, M. (2009). Historia urbana novohispánica del siglo XVI. Cd. de México, México: Universidad Nacional Autónoma de México, Facultad de Estudios Superiores de Acatlán.

de Sahagún, F. (2000). Historia general de las cosas de la Nueva España. México: CONACULTA.

Galindo Trejo, J., \& López, C. (2001). El cerro de San Miguel como posible marcador calendárico astronómico del sitio preclásico de Cuicuilco. En J. Broda, S. Iwaniszewski, \& A. Montero (Edits.), La montaña en el paisaje ritual (págs. 201-216). Cd. de México, México: Universidad Nacional Autónoma de México.

García Zambrano, Á. (2000). Antagonismos ideológicos de la urbanización temprana en la Nueva España. Estudios Históricos: Arquitectura y Diseño(5)

García Zambrano, Á. (2000). Antagonismos ideológicos de la urbanización temprana de la Nueva España. (No. 5), 21-42.

García Zambrano, Á. (2004). Pueblo Nuevo y Pueblo Viejo: recuperación de una memoria histórica. En 
L. Noelle (Ed.), La ciudad: problema integral de preservación patrimonial (Primera edición 2005 ed., págs. 59-82). Cd. de México, México: Universidad Nacional Autónoma de México, Instituto de Investigaciones Estéticas.

García Zambrano, Á. (2006). La construcción socio-histórica del paisaje fundacional en las migraciones mesoamericanas. En S. Miranda Pacheco, \& T. Lozano Armendares (Ed.), Espacio y sociedad en América: hacia una historia de sus reacciones (págs. 99-120). Sevilla: Universidad Nacional Autónoma de México.

García Zambrano, Á. (Junio de 2009). Frondas boscosas y parajes rocallosos: determinantes ambientales en los asentamientos indígenas de Mesoamérica colonial. VII(1), 3-13.

Kubler, G. (2009). Arquitectura Mexicana del Siglo XVI (Segunda edición en español 2012 ed.). Cd. de México, México: Fondo de Cultura Económica (FCE).

Ledesma Gallegos, L. (2012). Génesis de la arquitectura mendicante del siglo XVI en el plan de Amilpas y las cañadas de Morelos (Primera edición 2012 ed.). Cd. de México, México: Instituto Nacional de Antropología e Historia.

Lockhart, J. (1999). Los nahuas después de la conquista. Cd. de México: Fondo de Cultura Económica. López Austin, A. (1994). Tamoanchan y Tlalocan. Cd. de México, México: Fondo de Cultura

Económica.

López Austín, A., \& López Luján, L. (2001). El pasado indígena (Segunda edición 2001 ed.). Cd. de México, México: Fondo de Cultura Económica (FCE).

López Austin, A., \& Millones, L. (2008). Dioses del norte, Dioses del sur (Primera Edición ed.). Ciudad de México, México: Era.

McAndrew, J. (1965). The Open-Air Churches of Sixteenth-Century Mexico. Cambridge: Harvard University Press.
Montero García, I. A. (2001). Buscando a los Dioses de la montaña: una propuesta de clasificación ritual. En J. Broda, S. Iwaniszewski, \& A. Montero (Edits.), La montaña en el paisaje ritual (págs. 13- 22). Cd. de México, México: Universidad Nacional Autónoma de México.

Ponce de León H., A. (1983). Fechamiento arqueoastrónomico en el altiplano central de México.

Dirección general de planeación.

Robelo, C. (1912). Origen del Calendario Nahuatl. Apéndice, Reseña de la Segunda Sesión del XVII Congreso Internacional de Americanistas Efectuada en la Ciudad de México durante del Mes de

Septiembre de 1910 (págs. 9-21). Cd. de México: Imprenta del Museo de Arqueología, Historia y Etnología.

Smith, M. (1994). The size of the Aztec city of Yautepec, Urban survey in central Mexico. Ancient Mesoamerica(5), 1-11.

Smith, M. (1997). Achaeology in the Middle of Political Conflict in Yautepec, Mexico.

Smith, M. (2006). Excavaciones de casas postclasicas en la zona urbana de Yautepec, Morelos: informe final. 1-21: The digital archeological record.

Sprajc, I. (2001). Alineamientos astronómicos en Tenayuca, México. En J. Broda, S. Iwaniszewski, \&

A. Montero (Edits.), La montaña en el paisaje ritual. Cd. de México, México: Universidad Nacional Autónoma de México.

Sprajc, I. (2001). Orientaciones astronómicas en la arquitectura prehispánica del centro de México. Cd. de México, México: Instituto Nacional de Antropología e Historia (INAH), Consejo Nacional para la Cultura y las Artes (CONACULTA).

Weckmann, L. (1983:1996). La herencia medieval de México (Segunda Edición ed.). Cd. de México, México: Fondo de Cultura Económica. 